\title{
Compactification of moduli of Higgs bundles
}

\author{
Tamás Hausel \\ DPMMS, University of Cambridge
}

February 1, 2008

\begin{abstract}
In this paper we consider a canonical compactification of $\mathcal{M}$, the moduli space of stable Higgs bundles with fixed determinant of odd degree over a Riemann surface $\Sigma$, producing a projective variety $\overline{\mathcal{M}}=\mathcal{M} \cup Z$. We give a detailed study of the spaces $\overline{\mathcal{M}}, Z$ and $\mathcal{M}$. In doing so we reprove some assertions of Laumon and Thaddeus on the nilpotent cone.
\end{abstract}

\section{Introduction}

Magnetic monopoles, the solutions of Bogomolny equations of mathematical physics, can be interpreted as solutions of the self-dual Yang-Mills equations on $\mathbf{R}^{4}$ which are translation invariant in one direction. Motivated by this interpretation, Hitchin in Hit1 addressed the problem of finding solutions to the $S U(2)$ self-dual Yang-Mills equations on $\mathbf{R}^{4}$, which are translation invariant in two directions. Although such solutions of finite energy do not exist, due to the conformal invariance of the equations, it was possible to find solutions of the corresponding $S U(2)$ self-duality equations over a Riemann surface $\Sigma$. In the same paper Hitchin gave an extensive description of the space of these solutions.

One important result shows how to assign in a certain one to one manner an algebrogeometric object to a solution of Hitchin's self-duality equations. This algebro-geometric object is called a stable Higgs bundle, which consists of a pair of a rank 2 holomorphic vector bundle $E$ on $\Sigma$ and a section $\Phi \in H^{0}\left(\Sigma, \operatorname{End}_{0}(E) \otimes K_{\Sigma}\right)$. The latter is called the Higgs field, after the analogous object in the monopole case.

In [Hit], in these algebro-geometric terms, Hitchin investigates the moduli space $\mathcal{M}$ of stable Higgs bundles with fixed determinant of degree 1 . This notion and the corresponding moduli space has become important from a purely algebro-geometric point of view, too. The main reason is that the cotangent bundle of $\mathcal{N}$, the moduli space of stable rank 2 vector bundles with fixed determinant of odd degree, which is a well researched object in the algebraic geometry of vector bundles, sits inside $\mathcal{M}$ as an open dense subset. Namely, $\left(T_{\mathcal{N}}^{*}\right)_{E}$ is canonically isomorphic to $H^{0}\left(\Sigma, \operatorname{End}_{0}(E) \otimes K_{\Sigma}\right)$ thus the points of $T_{\mathcal{N}}^{*}$ are Higgs bundles. 
Among other results Hitchin proved that $\mathcal{M}$ is a non-compact complete hyperkähler manifold. Defined as above, in purely algebro-geometric terms, it was not surprising that $\mathcal{M}$ turned out to be quasi-projective as Nitsure has shown in $\mathbb{\text { Nit] }}$.

The main aim of this paper is to investigate a canonical compactification of $\mathcal{M}$ : among other things we show that the compactification is projective, calculate its Picard group, and calculate the Poincaré polynomial for the cohomology.

In this paper we use a simple method to compactify non-compact Kähler manifolds with a nice proper Hamiltonian $S^{1}$ action via Lerman's construction of symplectic cutting [Le]]. We use this method to compactify $\mathcal{M}$. Our approach is symplectic in nature and eventually produces some fundamental results about the spaces occurring, using existing techniques from the theory of symplectic quotients.

We show that the compactification described in this paper is a good example of Yau's problem of finding a complete Ricci flat metric on the complement of a nef anticanonical divisor in a projective variety.

Many of the results of this paper can be easily generalized to other Higgs bundle moduli spaces, which have been extensively investigated (see e.g. [Nit] and [Sim1]). As a matter of fact Simpson gave a definition of a similar compactificitation for these more general Higgs bundle moduli spaces in Theorems 11.2 and 11.1 of [Sim2 and in Proposition 17 of [Sim3], without investigating it in detail. For example, the projectiveness of the compactification is not clear from these definitions. One novelty of our paper is the proof of the projectiveness of the compactification in our case.

Since the compactification method used in this paper is fairly general it is possible to apply it to other Kähler manifolds with the above properties. It could be interesting for instance to see how this method works for the toric hyperkähler manifolds of Goto Goto and Bielawski and Dancer $B$ Bi,Da.

Finally, as a conclusion, we note that the compactification of this paper solves one half of the problem of compactifying the moduli space $\mathcal{M}$, namely the 'outer' half, i.e. shows what the resulting spaces look like; while the other half of the problem the 'inner' part, i.e. how this fits into the moduli space description of $\mathcal{M}$, is treated in the recent paper of Schmitt Schm. Schmitt's approach is algebro-geometric in nature, and concerns mainly the construction of the right notion for moduli to produce $\overline{\mathcal{M}}$, thus complements the present paper. The relation between the two approaches deserves further investigation.

Acknowledgements. First of all I would like to thank my supervisor Nigel Hitchin for fruitful supervisions. The compatibility with Yau's problem was suggested by Michael Atiyah, while Lerman's symplectic cutting was suggested by Michael Thaddeus. With both of them I had very inspiring conversations. I also thank Balázs Szendrői and the referee for helpful comments. Finally, I thank Trinity College, Cambridge for financial support.

\section{Statement of results}

In this section we describe the structure of the paper and list the results. 
In Section 3 we collect the existing results about $\mathbf{C}^{*}$ actions on Kähler manifolds and subsequently on Kähler quotients from the literature. We explain a general method of compactifying Kähler manifolds with a nice, proper, Hamiltonian $S^{1}$ action. The rest of the paper follows the structure of Section 3 .

In Section 1 we define the basic notions and restate some results of Hitchin about $\mathcal{M}$. Here we learn that the results of Section 3 apply to $\mathcal{M}$. We describe here our toy example $\mathcal{M}_{\text {toy }}$, the moduli space of parabolic Higgs bundles on $\mathbf{P}_{4}^{1}$, which serves as an example throughout the paper.

In Section 5 (following ideas of Subsection 3.1) we describe the nilpotent cone after Thaddeus [Tha1 and show that it coincides with the downward Morse flow (Theorem 5.2). We reprove Laumon's theorem in our case, that the nilpotent cone is Lagrangian (Corollary 5.3).

In Section 6 we describe $Z$, the highest level Kähler quotient of $\mathcal{M}$, while in 7 we analyse $\overline{\mathcal{M}}=\mathcal{M} \cup Z$. Here we follow the approaches of Subsection 3.2 and Subsection 3.3, respectively. Among others, we prove the following statements:

- $\overline{\mathcal{M}}$ is a compactification of $\mathcal{M}$, the moduli space of stable Higgs bundles with fixed determinant and degree 1 (Theorem 7.2).

- $Z$ is a symplectic quotient of $\mathcal{M}$ by the circle action $(E, \Phi) \mapsto\left(E, e^{i \theta} \Phi\right) . \overline{\mathcal{M}}$ is a symplectic quotient of $\mathcal{M} \times \mathbf{C}$ with respect to the circle action, which is the usual one on $\mathcal{M}$ and multiplication on $\mathbf{C}$.

- While $\mathcal{M}$ is a smooth manifold, $Z$ is an orbifold, with only $\mathbf{Z}_{2}$ singularities corresponding to the fixed point set of the map $(E, \Phi) \mapsto(E,-\Phi)$ on $\mathcal{M}$ (Theorem 6.2), while similarly $\overline{\mathcal{M}}$ is an orbifold with only $\mathbf{Z}_{2}$ singularities, and the singular locus of $\overline{\mathcal{M}}$ coincides with that of $Z$ (Theorem 7.3 ).

- The Hitchin map

$$
\chi: \mathcal{M} \rightarrow \mathbf{C}^{3 g-3}
$$

extends to a map

$$
\bar{\chi}: \overline{\mathcal{M}} \rightarrow \mathbf{P}^{3 g-3}
$$

which when restricted to $Z$ gives a map

$$
\bar{\chi}: Z \rightarrow \mathbf{P}^{3 g-4}
$$

whose generic fibre is a Kummer variety corresponding to the Prym variety of the generic fibre of the Hitchin map (Theorem 6.10, Theorem 7.8).

- $\overline{\mathcal{M}}$ is a projective variety (Theorem 7.11 ), with divisor $Z$ such that

$$
(3 g-2) Z=-K_{\overline{\mathcal{M}}},
$$

the anticanonical divisor of $\overline{\mathcal{M}}$ (Corollary 7.7 ). 
- Moreover, $Z$ itself is a projective variety (Theorem 6.16) with an inherited holomorphic contact structure with contact line bundle $L_{Z}$ (Theorem 6.9) and a oneparameter family of Kähler forms $\omega_{t}(Z)$ (Theorem 6.15). The Picard group of $Z$ is described in Corollary 6.7. Moreover, the normal bundle of $Z$ in $\overline{\mathcal{M}}$ is $L_{Z}$ which is nef by Corollary 6.14.

- Furthermore, $\overline{\mathcal{M}}$ has a one-parameter family of Kähler forms $\omega_{t}(\overline{\mathcal{M}})$, which when restricted $Z$ gives the above $\omega_{t}(Z)$.

- $Z$ is birationally equivalent to $P\left(T_{\mathcal{N}}^{*}\right)$ the projectivized cotangent bundle of the moduli space of rank 2 stable bundles with fixed determinant and odd degree (Corollary 6.4). $\overline{\mathcal{M}}$ is birationally equivalent to $P\left(T_{\mathcal{N}}^{*} \oplus \mathcal{O}_{\mathcal{N}}\right)$, the canonical compactification of $T_{\mathcal{N}}^{*}$ (Corollary 7.4).

- We calculate certain sheaf cohomology groups in Corollary 6.12 and Corollary 6.13 and interpret some of these results as the equality of certain infinitesimal deformation spaces.

- The Poincaré polynomial of $Z$ is described in Corollary 6.5, the Poincaré polynomial of $\overline{\mathcal{M}}$ is described in Theorem 7.12 .

- We finish Section 7 by showing an interesting isomorphism between two vector spaces: one contains information about the intersection of the components of the nilpotent cone, the other says something about the contact line bundle $L_{Z}$ on $Z$.

\section{Compactification by symplectic cutting}

In this section we collect the results from the literature concerning $\mathbf{C}^{*}$ actions on Kähler manifolds. At the same time we sketch the structure of the rest of the paper.

\subsection{Stratifications}

Suppose that we are given a Kähler manifold $(M, I, \omega)$ with complex structure $I$ and Kähler form $\omega$. Suppose also that $\mathbf{C}^{*}$ acts on $M$ biholomorphically with respect to $I$ and such that the Kähler form is invariant under the induced action of $S^{1} \subset \mathbf{C}^{*}$. Suppose furthermore that this latter action is Hamiltonian with proper moment map $\mu: M \rightarrow \mathbf{R}$, with finitely many critical points and 0 being the absolute minimum of $\mu$. Let $\left\{N_{\alpha}\right\}_{\alpha \in A}$ be the set of the components of the fixed point set of the $\mathbf{C}^{*}$ action.

We list some results of [Kir] extended to our case. Namely, Kirwan's results are stated for compact Kähler manifolds, but one can always modify the proofs for non-compact manifolds as above (cf. Chapter 9 in [Kir]).

There exist two stratifications in such a situation. The first one is called the Morse stratification and can be defined as follows. The stratum $S_{\alpha}^{M}$ is the set of points of $\mathcal{M}$ whose path of steepest descent for the Morse function $\mu$ and the Kähler metric have limit points in $N_{\alpha}$. One can also define the sets $T_{\alpha}^{M}$ as the points of $\mathcal{M}$ whose path of steepest 
descent for the Morse function $-\mu$ and the Kähler metric have limit points in $N_{\alpha}$. $S_{\alpha}^{M}$ gives a stratification even in the non-compact case, however the set $\bigcup_{\alpha} T_{\alpha}^{M}$ is not the whole space but a deformation retract of it. The set $\bigcup_{\alpha} T_{\alpha}^{M}$ is called the downward Morse flow.

The other stratification is the Bialynicki-Birula stratification, where the stratum $S_{\alpha}^{B}$ is the set of points $p \in M$ for which $\lim _{t \rightarrow 0} t p \in N_{\alpha}$. Similarly, as above, we can define $T_{\alpha}^{B}$ as the points $p \in M$ for which $\lim _{t \rightarrow \infty} t p \in N_{\alpha}$.

One of Kirwan's important results in [Kin Theorem 6.16 asserts that the stratifications $S_{\alpha}^{M}$ and $S_{\alpha}^{B}$ coincide, and similarly $T_{\alpha}^{M}=T_{\alpha}^{B}=T_{\alpha}$. This result is important because it shows that the strata $S_{\alpha}=S_{\alpha}^{M}=S_{\alpha}^{B}$ of the stratifications are total spaces of affine bundles (so-called $\beta$-fibrations) on $N_{\alpha}$ (this follows from the Bialynicki-Birula picture) and moreover this stratification is responsible for the topology of the space $M$ (this follows from the Morse picture). Thus we have the following theorem (cf. Theorem 4.1 of [Bia] and also Theorem 1.12 of Tha3):

Theorem $3.1 S_{\alpha}$ and $T_{\alpha}$ are complex submanifolds of $M$. They are isomorphic to total spaces of some $\beta$-fibrations over $N_{\alpha}$, such that the normal bundle of $N_{\alpha}$ in these $\beta$ fibrations are $E_{\alpha}^{+}$and $E_{\alpha}^{-}$, respectively, where $E_{\alpha}^{+}$is the positive and $E_{\alpha}^{-}$is the negative subbundle of $\left.T_{M}\right|_{N_{\alpha}}$ with respect to the $S^{1}$ action.

Moreover, the downward Morse flow $\bigcup_{\alpha} T_{\alpha}$ is a deformation retract of $M$.

Recall that a $\beta$-fibration in our case is a fibration $E \rightarrow B^{n}$ with a $\mathbf{C}^{*}$ action on the total space which is locally like $\mathbf{C}^{n} \times V$, where $V$ is the $\mathbf{C}^{*}$ module $\beta: \mathbf{C}^{*} \rightarrow G L(V)$. Note that such a fibration is not a vector bundle in general, but it is if $\beta$ is the sum of isomorphic, one-dimensional non-trivial $\mathbf{C}^{*}$ modules.

\subsection{Kähler quotients}

We define an action to be semi-free if the stabilizer of any point is finite or the whole group itself.

Whenever we are given a Hamiltonian, proper, semi-free $S^{1}$ action on a Kähler manifold, we can form the Kähler quotients $Q_{t}=\mu^{-1}(t) / S^{1}$, which are compact Kähler orbifolds at a regular value $t$ of $\mu$.

If this $S^{1}$ action is induced from an action of $\mathbf{C}^{*}$ on $M$ as above, then we can relate the Kähler quotients to the quotients $M / \mathbf{C}^{*}$ as follows. First we define $M_{t}^{\min } \subset M$ as the set of points in $M$ whose $\mathbf{C}^{*}$ orbit intersects $\mu^{-1}(t)$. Now a theorem of Kirwan states (see Theorem 7.4 in [Kir]) that it is possible to define a complex structure on the orbit space $M_{t}^{\min } / \mathbf{C}^{*}$, and she also proves that this space is homeomorphic to $Q_{t}$, defining the complex structure for the Kähler quotient $Q_{t}$. (Here again we used the results of Kirwan for non-compact manifolds, but as above, these results can be easily modified for our situation.) It now simply follows that $M_{t}^{\text {min }}$ only depends on that connected component of the regular values of $\mu$ in which $t$ lies, and as a consequence of this we can see that the complex structure on $Q_{t}$ is the same as on $Q_{t^{\prime}}$ if the interval $\left[t, t^{\prime}\right]$ does not contain any critical value of $\mu$. We have as a conclusion the following theorem:

Theorem 3.2 At a regular level $t \in \mathbf{R}$ of the moment map $\mu$, we have the Kähler quotient $Q_{t}=\mu^{-1}(t) / S^{1}$ which is a compact Kähler orbifold with $M_{t}^{\text {min }}$ as a holomorphic $\mathbf{C}^{*}$ 
principal orbibundle on it. Moreover $M_{t}^{\text {min }}$ and the complex structure on $Q_{t}$ only depend on that connected component of the regular values of $\mu$ where $t$ lies.

It follow from the above theorem that there is a discrete family of complex orbifolds which arise from the above construction. Moreover, at each level we get a Kähler form on the corresponding complex orbifold. The evolution of the different Kähler quotients has been well investigated (e.g. in the papers $\mathrm{Du}, \mathrm{He}$, Gu,St, cf also Tha3 and Br,Pr]). We can summarize these results in the following theorem:

Theorem 3.3 The Kähler quotients $Q_{t}$ and $Q_{t^{\prime}}$ are biholomorphic if the interval $\left[t, t^{\prime}\right]$ does not contain a critical value of the moment map. They are related by a blowup followed by a blow-down if the interval $\left[t, t^{\prime}\right]$ contains exactly one critical point $c$ different from the endpoints. To be more precise, $Q_{t}$ blown up along the union of submanifolds $\bigcup_{\mu\left(N_{\alpha}\right)=c} P_{w}\left(E_{\alpha}^{-}\right)$is isomorphic to $Q_{t^{\prime}}$ blown up along $\bigcup_{\mu\left(N_{\alpha}\right)=c} P_{w}\left(E_{\alpha}^{+}\right)$and in both cases the exceptional divisor is $\bigcup_{\mu\left(N_{\alpha}\right)=c} P_{w}\left(E_{\alpha}^{+}\right) \times_{N_{\alpha}} P_{w}\left(E_{\alpha}^{-}\right)$the fibre product of weighted projective bundles over $N_{\alpha}$.

Moreover, in a connected component of the regular values of $\mu$ the cohomology classes of the Kähler forms $\omega_{t}\left(Q_{t}\right)$ depend linearly on $t$ according to the formula:

$$
\left[\omega_{t}\left(Q_{t}\right)\right]-\left[\omega_{t^{\prime}}\left(Q_{t^{\prime}}\right)\right]=\left(t-t^{\prime}\right) c_{1}\left(M_{t}^{\min }\right)=\left(t-t^{\prime}\right) c_{1}\left(M_{t^{\prime}}^{\min }\right)
$$

where $c_{1}$ is the first Chern class of the $\mathbf{C}^{*}$ principal bundle.

\subsection{Symplectic cuts}

Now let us recall the construction of the symplectic cut we need (see [Ler] and also Ed,Gr for the algebraic case), first in the symplectic and second in the Kähler category.

If $(M, \omega)$ is a symplectic manifold with a Hamiltonian and semi-free $S^{1}$ action and proper moment map $\mu$ with absolute minimum 0 , then we can define the symplectic cut of $M$ at the regular level $t$ by a symplectic quotient construction as follows.

We let $S^{1}$ act on the symplectic manifold $M \times \mathbf{C}$ (where the symplectic structure is the product of the symplectic structure on $M$ and the standard symplectic structure on C) by acting on the first factor according to the above $S^{1}$ action and on the second factor by the standard multiplication. This action is clearly Hamiltonian with proper moment map $\mu+\mu_{\mathbf{C}}$, where $\mu_{\mathbf{C}}$ is the standard moment map on $\mathbf{C}: \mu_{C}(z)=|z|^{2}$.

Now if $t$ is a regular value of the moment map $\mu+\mu_{\mathbf{C}}$, such that $S^{1}$ acts with finite stabilizers on $M_{t}=\mu^{-1}(t)$ (i.e. $M_{t} / S^{1}$ gives a symplectic orbifold), then the symplectic quotient $\bar{M}_{\mu<t}$ defined by

$$
\bar{M}_{\mu<t}=\left\{(m, w) \in M \times \mathbf{C}: \mu(m)+|w|^{2}=t\right\}
$$

will be a symplectic compactification of the symplectic manifold $M_{\mu<t}$ in the sense that

$$
\bar{M}_{\mu<t}=M_{\mu<t} \cup Q_{t},
$$


and the inherited symplectic structure on $\bar{M}_{\mu<t}$ restricted to $M_{\mu<t}$ coincides with its original symplectic structure. Moreover, if we restrict this structure onto $Q_{t}$ it coincides with its quotient symplectic structure.

Now suppose that we are given a Kähler manifold $(M, I, \omega)$ and a holomorphic $\mathbf{C}^{*}$ action on it, such that the induced $S^{1} \subset \mathbf{C}^{*}$ action preserves the Kähler form and is semi-free and Hamiltonian with proper moment map. With these extra structures the symplectic cut construction will give us $\bar{M}_{\mu<t}$ a compact Kähler orbifold with a $\mathbf{C}^{*}$ action, such that $\bar{M}_{\mu<t} \backslash Q_{t}$ is symplectomorphic to $M_{\mu<t}$ as above and furthermore is biholomorphic to $\mathbf{C}^{*}\left(M_{\mu<t}\right)$, the union of $\mathbf{C}^{*}$-orbits intersecting $M_{\mu<t}$. (This is actually an important point, as it shows that $M_{\mu<t}$ is not Kähler isomorphic to $\bar{M}_{\mu<t} \backslash Q_{t}$, cf [Ler]). We can collect all these results into the next theorem:

Theorem 3.4 The symplectic cut $\bar{M}_{\mu<t}=M_{\mu<t} \cup Q_{t}$ as a symplectic manifold compactifies the symplectic manifold $M_{\mu<t}$, such that the restricted symplectic structure on $Q_{t}$ coincides with the quotient symplectic structure.

Furthermore if $M$ is a Kähler manifold with a $\mathbf{C}^{*}$ action as above, then $\bar{M}_{\mu<t}$ will be a Kähler orbifold with a $\mathbf{C}^{*}$ action, such that $Q_{t}$ with its quotient complex structure is a codimension 1 complex suborbifold of $\bar{M}_{\mu<t}$ whose complement is equivariantly biholomorphic to $\mathbf{C}^{*}\left(M_{\mu<t}\right)$ with its canonical $\mathbf{C}^{*}$ action.

Remark. Note that if $t$ is higher than the highest critical value (this implies that we have finitely many of them), then $\mathbf{C}^{*}\left(M_{\mu<t}\right)=M$ is the whole space, therefore the symplectic cutting in this case gives a holomorphic compactification of $M$ itself. The compactification is $\bar{M}_{\mu<t}$, which is equal to the quotient of $(M \times \mathbf{C}-N \times\{0\})$ by the action of $\mathbf{C}^{*}$, where $N$ is the downward Morse flow. This is the compactification we shall examine here for the case of $\mathcal{M}$, the moduli space of stable Higgs bundles with fixed determinant of degree 1.

\section{The moduli of Higgs bundles $\mathcal{M}$}

Notation 4.1 Let

- $\Sigma$ be a closed Riemann surface of genus $g>1$,

- $\Lambda$ a fixed line bundle on $\Sigma$ of degree 1 ,

- $\mathcal{N}$ the moduli space of rank 2 stable bundles with determinant $\Lambda$,

- $\mathcal{M}$ denote the moduli space of stable Higgs pairs $(E, \Phi)$, where $E$ is a rank 2 vector bundle on $\Sigma$ with $\operatorname{det} E=\Lambda$ and $\Phi \in H^{0}\left(\Sigma, E n d_{0} E \otimes K_{\Sigma}\right)$.

Remark. For the terms used above we refer the reader to [Hit] and [Sim1].

After introducing the space $\mathcal{M}$, Hitchin gave its extensive description in [Hit], [Hit2]. Here we restate some of his results. 
- $\mathcal{M}$ is a noncompact, smooth manifold of dimension $12 g-12$ containing $T_{\mathcal{N}}^{*}$ as a dense open set.

- Furthermore $\mathcal{M}$ is canonically a Riemannian manifold with a complete hyperkähler metric. Thus $\mathcal{M}$ has complex structures parameterized by $S^{2}$. One of the complex structures, for which $T_{\mathcal{N}}^{*}$ is a complex submanifold, is distinguished, call it $I$. We will only be concerned with this complex structure here. The others (apart from $-I$ ) are biholomorphic to each other and give $\mathcal{M}$ the structure of a Stein manifold. From these Kähler forms one can build up a holomorphic symplectic form $\omega_{h}$ on $(\mathcal{M}, I)$.

- There is a map, called the Hitchin map

$$
\chi: \mathcal{M} \rightarrow H^{0}\left(\Sigma, K_{\Sigma}^{2}\right)=\mathbf{C}^{3 g-3}
$$

defined by

$$
(E, \Phi) \mapsto \operatorname{det} \Phi
$$

The Hitchin map is proper and an algebraically completely integrable Hamiltonian system with respect to the holomorphic symplectic form $\omega_{h}$, with generic fibre a Prym variety corresponding to the spectral cover of $\Sigma$ at the image point.

- Let $\omega$ denote the Kähler form corresponding to the complex structure $I$. There is a holomorphic $\mathbf{C}^{*}$ action on $\mathcal{M}$ defined by $(E, \Phi) \mapsto(E, z \Phi)$. The restricted action of $S^{1}$ defined by $(E, \Phi) \rightarrow\left(E, e^{i \theta} \Phi\right)$ is isometric and indeed Hamiltonian with proper moment map $\mu$. The function $\mu$ is a perfect Morse function, moreover:

$\mu$ has $g$ critical values: an absolute minimum $c_{0}=0$ and $c_{d}=\left(d-\frac{1}{2}\right) \pi$, where $d=1, \ldots, g-1$.

$\mu^{-1}\left(c_{0}\right)=\mu^{-1}(0)=N_{0}=\mathcal{N}$ is a non-degenerate critical manifold of index 0 .

$\mu^{-1}\left(c_{d}\right)=N_{d}$ is a non-degenerate critical manifold of index $2(g+2 d-2)$ and is diffeomorphic to a $2^{2 g}$-fold cover of the $(2 g-2 d-1)$-fold symmetric product $S^{2 g-2 d-1}(\Sigma)$.

- The fixed point set $S$ of the involution $\sigma(E, \Phi)=(E,-\Phi)$ is the union of $g$ complex submanifolds of $\mathcal{M}$ namely,

$$
S=\mathcal{N} \cup \bigcup_{d=1}^{g-1} F_{d},
$$

where $F_{d}$ is the total space of a vector bundle $F_{d}$ over $Z_{d}$. Moreover $F_{d}$ is a complex submanifold of dimension $3 g-3$.

Using an algebraic point of view Nitsure in Nit could prove:

Theorem 4.2 (Nitsure) $\mathcal{M}$ is a quasi-projective variety.

The main aim of this paper is to examine in certain sense the canonical compactification of $\mathcal{M}$. 
Example. Unfortunately, even when $g=2$ the moduli space $\mathcal{M}$ is already 6 dimensional, too big to serve as an instructive example. We rather choose $\mathcal{M}_{\text {toy }}$, the moduli space of stable parabolic Higgs bundles on $\mathbf{P}^{1}$, with four marked points, in order to show how our later constructions work. (These moduli spaces were considered by Yokogawa Yoko.) We choose this example because it is a complex surface, and can be constructed explicitly.

We fix four distinct points on $\mathbf{P}^{1}$ and denote by $\mathbf{P}_{4}^{1}$ the corresponding complex orbifold. Let $P$ be the elliptic curve corresponding to $\mathbf{P}_{4}^{1}$. Let $\sigma_{P}$ be the involution $\sigma_{P}(x)=-x$ on $P$. Thus, $P / \sigma_{P}$ is just the complex orbifold $\mathbf{P}_{4}^{1}$. The four fixed points of the involution $x_{1}, x_{2}, x_{3}, x_{4} \in P$ correspond to the four marked points on $\mathbf{P}_{4}^{1}$. Furthermore, let $\tau$ be the involution $\tau(z)=-z$ on $\mathbf{C}$.

Consider now the quotient space $(P \times \mathbf{C}) /\left(\sigma_{P} \times \tau\right)$. This is a complex orbifold of dimension 2 with four isolated $\mathbf{Z}_{2}$ quotient singularities at the points $x_{i} \times 0$. Blowing up these singularities we get a smooth complex surface $\mathcal{M}_{\text {toy }}$ with four exceptional divisors $D_{1}, D_{2}, D_{3}$ and $D_{4}$. Moreover the map $\chi:(P \times \mathbf{C}) \rightarrow \mathbf{C}$ sending $(z, x) \mapsto z^{2}$, descends to the quotient $(P \times \mathbf{C}) /\left(\sigma_{P} \times \tau\right)$ and sending the exceptional divisors to zero one obtains a map $\chi_{\text {toy }}: \mathcal{M}_{\text {toy }} \rightarrow \mathbf{C}$, with generic fibre $P$. The map $\chi_{\text {toy }}$ will serve as our toy Hitchin map.

Moreover there is a $\mathbf{C}^{*}$ action on $\mathcal{M}_{\text {toy }}$, coming from the standard action on $\mathbf{C}$. The fixed point set of $S^{1} \subset \mathrm{C}^{*}$ has five components: one is $\mathcal{N}_{\text {toy }} \subset \mathcal{M}_{\text {toy }}$ (the moduli space of stable parabolic bundles on $\left.\mathbf{P}_{4}^{1}\right)$ which is the proper transform of $(P \times 0) /\left(\tau \times \sigma_{P}\right)=$ $P_{4}^{1} \subset(P \times \mathbf{C}) /\left(\sigma_{P} \times \tau\right)$ in $\mathcal{M}_{\text {toy }}$. The other four components consist of single points $\tilde{x}_{i} \in D_{i}, i=1,2,3,4$.

The fixed point set of the involution $\sigma: \mathcal{M}_{\text {toy }} \rightarrow \mathcal{M}_{\text {toy }}$ has five components, one of which is $\mathcal{N}_{\text {toy }}$, the others $F_{i}$ are the proper transforms of the sets $\left(x_{i} \times \mathbf{C}\right) /\left(\sigma_{P} \times \tau\right) \subset$ $(P \times \mathbf{C}) /\left(\sigma_{P} \times \tau\right)$.

\section{The nilpotent cone $N$}

The results in the previous section show that the Kähler manifold $(\mathcal{M}, I, \omega)$ is equipped with a $\mathbf{C}^{*}$ action which restricts to an $S^{1} \subset \mathbf{C}^{*}$ action which is semi-free and Hamiltonian with proper moment map $\mu$. Moreover, 0 is an absolute minimum for $\mu$. Therefore we are in the situtation described in Section 3. In the following sections we will apply the ideas developed there to our situation and deduce important properties of the spaces $\mathcal{M}$, $Z$ and $\overline{\mathcal{M}}$.

We saw in Theorem 3.1 that the downward Morse flow is a deformation retract of $\mathcal{M}$, so it is responsible for the topology, and as such it is an important object. On the other hand we will prove that the downward Morse flow coincides with the nilpotent cone.

Definition 5.1 The nilpotent cone is the preimage of zero of the Hitchin map $N=$ $\chi^{-1}(0)$.

The name 'nilpotent cone' was given by Laumon, to emphasize the analogy with the nilpotent cone in a Lie algebra. 
In our context this is the most important fibre of the Hitchin map, and the most singular one at the same time. We will show below that the nilpotent cone is a central notion in our considerations.

Laumon in Lau investigated the nilpotent cone in a much more general context and showed its importance in the Geometric Langlands Correspondence. Thaddeus in [Tha1 concentrated on our case, and gave the exact description of the nilpotent cone. In what follows we will reprove some of their results.

The following assertion was already stated in [Tha1] which will turn out to be crucial in some of our considerations.

Theorem 5.2 The downward Morse flow coincides with the nilpotent cone.

Proof. As we saw in Theorem 3.1 the downward Morse flow can be identified with the set of points in $\mathcal{M}$ whose $\mathbf{C}^{*}$ orbit is relatively compact in $\mathcal{M}$.

Since the nilpotent cone is invariant under the $\mathbf{C}^{*}$ action and compact ( $\chi$ is proper) we immediately get that the nilpotent cone is a subset of the downward Morse flow.

On the other hand if a point in $\mathcal{M}$ is not in the nilpotent cone then the image of its $\mathbf{C}^{*}$ orbit by the Hitchin map is a line in $\mathbf{C}^{3 g-3}$, therefore cannot be relatively compact.

Laumon's main result is the following assertion (cf. Theorem 3.1 in [Lau]), which we prove in our case:

Corollary 5.3 (Laumon) The nilpotent cone is a Lagrangian subvariety of $\mathcal{M}$ with respect to the holomorphic symplectic form $\omega_{h}$.

Proof. The Hitchin map is a completely integrable Hamiltonian system, and the nilpotent cone is a fibre of this map, so it is coisotropic. Therefore it is Lagrangian if and only if its dimension is $3 g-3$.

On the other hand the nilpotent cone is exactly the downward Morse flow and we can use Hitchin's description of the critical submanifolds in [Hit], giving that the sum of the index and the real dimension of any critical submanifold is $6 g-6$. We therefore conclude that the complex dimension of the downward Morse flow (i.e. the nilpotent cone) is $3 g-3$.

Remark. Nakajima's Proposition 7.1 in Nak states that if $X$ is a Kähler manifold with a $\mathbf{C}^{*}$ action and a holomorphic symplectic form $\omega_{h}$ of homogeneity 1 then the downward Morse flow of $X$ is Lagrangian with respect to $\omega_{h}$. Thus Nakajima's result and Theorem 5.2 together give an alternative proof of the theorem. We prefered the one above for it concentrates on the specific properties of $\mathcal{M}$.

From the above proof we can see that for higher rank Higgs bundles Laumon's theorem is equivalent to the assertion that every critical submanifold contributes to the middle dimensional cohomology, i.e the sum of the index and the real dimension of any critical submanifold should always be half of the real dimension of the corresponding moduli space. 
Using the results of Goth1 one easily shows that the above statement also holds for the rank 3 case. Gothen could show directly the above statement for any rank and therefore gave an alternative proof of Laumon's theorem in these cases [Goth2.

Corollary 5.4 The middle dimensional homology $H_{6 g-6}(\mathcal{M})$ of $\mathcal{M}$ is freely generated by the homology classes of components of the nilpotent cone and therefore has dimension $g$.

Proof. We know that each component of $N$ is a projective variety of dimension $3 g-3$. $N$ is a deformation retract of $\mathcal{M}$, therefore the middle dimensional homology of $\mathcal{M}$ is generated by the homology classes of the components of $N$. Furthemore, from the Morse picture, components of $N$ are in a one to one correspondence with the critical manifolds of $\mathcal{M}$, so there are $g$ of them. The result follows.

We finish this section with Thaddeus's description of the nilpotent cone (see [Tha1, cf. [Lau]).

Theorem 5.5 The nilpotent cone is the union of $\mathcal{N}$ and the total spaces of vector bundles $E_{d}^{-}$over $N_{d}$, where $E_{d}^{-}$is the negative subbundle of $\left.T_{\mathcal{M}}\right|_{N_{d}}$.

Moreover, the restricted action of $\mathbf{C}^{*}$ on $N$ is just the inverse multiplication on the fibres.

Proof. This follows directly from Theorem 3.1 and Theorem 5.2, with noting that by Hitchin's description of the weights of the circle action on $T_{\mathcal{M}} \mid N_{d}$ in the proof of Proposition 7.1 of [Hit]], we have that there is only one negative weight. Therefore the $\beta$-fibration of Theorem 3.1 is a vector bundle in this case. The result follows.

Remark. From the description of $E_{d}^{-}$in Tha1 and that of $F_{d}$, a component of the fixed point set of the involution $\sigma(E, \Phi)=(E,-\Phi)$, in [Hit], one obtains the remarkable fact that the vector bundle $E_{d}^{-}$is actually dual to $F_{d}$.

Example. In our toy example we have the elliptic fibration $\chi_{\text {toy }}: \mathcal{M}_{\text {toy }} \rightarrow \mathbf{C}$, with the only singular fibre $N_{\text {toy }}=\chi_{\text {toy }}^{-1}(0)$, the toy nilpotent cone. We have now the decomposition

$$
N_{\text {toy }}=\mathcal{N}_{\text {toy }} \cup \bigcup_{i=1}^{4} D_{i}
$$

where we think of $D_{i}$ as the closure of $E_{i}$, the total space of the trivial line bundle on $\tilde{x}_{i}$.

The possible singular fibres of elliptic fibrations have been classified by Kodaira (cf. B,P,V, p. 150). According to this classification $N_{\text {toy }}$ is of type $I_{0}^{*}\left(\tilde{D}_{4}\right)$.

\section{The highest level Kähler quotient $Z$}

In this section we apply the ideas of Subsection 3.2 to our situation. 
Definition 6.1 Define for every non negative t the Kähler quotient

$$
Q_{t}=\mu^{-1}(t) / S^{1}
$$

As the complex structure of the Kähler quotient depends only on the connected component of the regular values of $\mu$, we can define $Z_{d}=Q_{t}$ for $c_{d}<t<c_{d+1}$ as a complex orbifold (we take $\left.c_{g}=\infty\right)$. Similarly, we define $X_{Z_{d}}$ to be $\mathcal{M}_{t}^{\text {min }}$ for $c_{d}<t<c_{d+1}$.

For simplicity let the highest level quotient $Z_{g-1}$ be denoted by $Z$ and the corresponding C $^{*}$ principal bundle $X_{Z_{g-1}}$ by $X_{Z}$.

In the spirit of Theorem 3.3 we have the following

Theorem 6.2 $Z_{d}$ is a complex orbifold with only $\mathbf{Z}_{2}$-singularities, where the singular locus is diffeomorphic to some union of projectivized vector bundles $P\left(F_{i}\right)$ :

$$
\operatorname{sing}\left(Z_{d}\right)=\bigcup_{0<i \leq d} P\left(F_{i}\right),
$$

where $F_{i} \subset \mathcal{M}$ is the total space of a vector bundle over $N_{i}$ and is a component of the fixed point set of the involution $\sigma(E, \Phi)=(E,-\Phi)$.

Proof. The induced action of $S^{1}$ on $\mathbf{C}^{3 g-3}$ by the Hitchin map is multiplication by $e^{2 i \theta}$ so an orbit of $S^{1}$ on $\mathcal{M} \backslash N$ is a non trivial double cover of the image orbit on $\mathbf{C}^{3 g-3}$. On the other hand by Thaddeus' description of $N$ (Theorem 5.5) it is clear that if a point of $N$ is not a fixed point of the circle action, then the stabilizer is trivial at that point.

Summarizing these two observations we obtain that if a point of $\mathcal{M}$ is not fixed by $S^{1}$, then its stabilizer is either trivial or $\mathbf{Z}_{2}$. The latter case occurs exactly at the fixed point set of the involution $\sigma$. The statement now follows from Theorem 3.3.

Proposition 6.3 $Z_{d}$ and $Z_{d+1}$ are related by a blowup following by a blowdown.

Namely, $Z_{d}$ blown up along $P\left(E_{d}^{-}\right)$is the same as the singular quotient $Q_{c_{d}}$ blown up along $N_{d}$ (its singular locus), which in turn gives $Z_{d+1}$ blown up at $P\left(E_{d}^{+}\right)$.

Moreover, this birational equivalence is an isomorphism outside an analytic set of codimension at least 3 .

Proof. The first bit is just the restatement of Theorem 3.3 in our setting.

The second part follows because $\operatorname{dim}\left(P\left(E_{d}^{-}\right)\right)=3 g-3-1<6 g-6-2$ and $\operatorname{dim}\left(P\left(E_{d}^{+}\right)\right)=3 g-3+2 g-2 d-1-1<6 g-6-2$ for $g>1$.

Corollary 6.4 $Z=Z_{g-1}$ is birationally equivalent to $P\left(T_{\mathcal{N}}^{*}\right)=Z_{0}$. Moreover this gives an isomorphism in codimension $>2$. 
Proof. Obviously $X_{Z_{0}}$ is $T_{\mathcal{N}}^{*}$, and therefore by Theoreom $3.2 Z_{0}$ is isomorphic to the projectivized cotangent bundle $P\left(T_{\mathcal{N}}^{*}\right)$. The statement follows from the previous theorem.

Corollary 6.5 Z has Poincaré polynomial

$$
P_{t}(Z)=\frac{t^{6 g-6}-1}{t^{2}-1} P_{t}(\mathcal{N})+\sum_{i=1}^{g-1} \frac{t^{6 g-6}-t^{2 g-4+4 i}}{t^{2}-1} P_{t}\left(N_{i}\right),
$$

where $N_{i}$ is a $2^{2 g}$-fold cover of $S^{2 i-1} \Sigma$.

Proof. One way to derive this formula is through Kirwan's formula in [Kir]. We use the above blowup, blowdown picture instead. This approach is due to Thaddeus, see [Tha2].

Applying the formula in [Gr,Ha],p.605 twice we get that

$$
P_{t}\left(Z_{d+1}\right)-P_{t}\left(Z_{d}\right)=P_{t}\left(P E_{d}^{+}\right)-P_{t}\left(P E_{d}^{-}\right)
$$

On the other hand for a projective bundle on a manifold $P \rightarrow M$ with fiber $\mathbf{P}^{n}$ one has (cf. Gr,Ha p.606)

$$
P_{t}(P)=\frac{t^{2 n+2}-1}{t^{2}-1} P_{t}(M)
$$

Hence the formula follows.

Remark. All the Poincaré polynomials on the right hand side of the above formula have been calculated. For $P_{t}(\mathcal{N})$ see e.g. [At,Bo for $P_{t}\left(N_{d}\right)$ see [Hit1].

We will determine the Picard group of $Z$ exactly. First we define some line bundles on several spaces.

Notation 6.6 Let

- $\mathcal{L}_{\mathcal{N}}$ denote the ample generator of the Picard group of $\mathcal{N}$ (cf. Dr,Na)

- $\mathcal{L}_{P T_{\mathcal{N}}^{*}}$ be its pullback to $P T_{\mathcal{N}}^{*}$

- $\mathcal{L}_{Z}$ denote the corresponding line bundle on $Z$ (cf. Corollary 6.4).

- $L_{P T_{\mathcal{N}}^{*}}$ be the dual of the tautological line bundle on $P T_{\mathcal{N}}^{*}$,

- $L_{Z}=X_{Z}^{*} \times_{\mathbf{C}^{*}} \mathbf{C}$ denote the corresponding line orbibundle on $Z$.

Corollary 6.7 $\operatorname{Pic}(Z)$, the Picard group of $Z$, is of rank 2 over $\mathbf{Z}$ and is freely generated by $\mathcal{L}_{Z}$ and $L_{Z}$. 
Remark. The Picard group of $Z$ is the group of invertible sheaves on $Z$. As the singular locus of $Z$ has codimension $\geq 2$, this group can be thought of as the group of holomorphic line orbibundles on $Z$. Namely, in this case the restriction of a holomorphic line orbibundle to $Z \backslash \operatorname{sing}(Z)$ gives a one-to-one correspondence between holomorphic line orbibundles on $Z$ and holomorphic line bundles on $Z \backslash \operatorname{sing}(Z)$, by the approriate version of Hartog's theorem.

Proof. It is well known that $\operatorname{Pic}(\mathcal{N})$ is freely generated by one ample line bundle $\mathcal{L}_{\mathcal{N}}$ therefore is of rank 1 (cf. [Dr,Na]). Thus $\operatorname{Pic}\left(P\left(T_{\mathcal{N}}^{*}\right)\right)$ is of rank 2 and freely generated by $\mathcal{L}_{P T_{\mathcal{N}}^{*}}$ the pullback of $\mathcal{L}_{\mathcal{N}}$ and the dual of the tautological line bundle $L_{P T_{\mathcal{N}}^{*}}$. From Corollary 6.4 $\operatorname{Pic}(Z)$ is isomorphic with $\operatorname{Pic}\left(P\left(T_{\mathcal{N}}^{*}\right)\right)$ therefore is of rank 2, and freely generated by $\mathcal{L}_{Z}$ and $L_{Z}$, where $\mathcal{L}_{Z}$ is isomorphic to $\mathcal{L}_{P T_{\mathcal{N}}^{*}}$ and $L_{Z}$ is isomorphic to $L_{P T_{\mathcal{N}}^{*}}$ outside the codimension 2 subset of Corollary 6.4.

Definition 6.8 A contact structure on a compact complex orbifold $Z$ of complex dimension $2 n-1$ is given by the following data:

1. a contact line orbibundle $L_{Z}$ such that $L_{Z}^{n}=K_{Z}^{-1}$, where $K_{Z}$ is the line orbibundle of the canonical divisor of $Z$,

2. a complex contact form $\theta \in H^{0}\left(Z, \Omega^{1}(Z) \otimes L_{Z}\right)$ a holomorphic $L_{Z}$ valued 1-form, such that

$$
0 \neq \theta \wedge(d \theta)^{n-1} \in H^{0}\left(Z, \Omega^{2 n-1}(Z) \otimes K_{Z}^{-1}\right)=H^{0}\left(Z, \mathcal{O}_{Z}\right)=\mathbf{C}
$$

is a nonzero constant.

Theorem 6.9 There is a canonical holomorphic contact structure on $Z$ with contact line orbibundle $L_{Z}$.

Proof. This contact structure can be created by the construction of Lebrun as in Leb] Remark 2.2. We only have to note that the holomorphic symplectic form $\omega_{h}$ on $\mathcal{M}$ is of homogeneity 1.

The construction goes as follows. If $\pi: X_{Z}^{*} \rightarrow Z$ denotes the canonical projection of the $\mathbf{C}^{*}$ principal orbibundle $X_{Z}^{*}$ the dual of $X_{Z}$, then $\pi^{*}\left(L_{Z}\right)$ is canonically trivial with the canonical section having homogeneity 1 . Thus in order to give a complex contact form $\theta \in H^{0}\left(Z, \Omega^{1}(Z) \otimes L_{Z}\right)$ it is sufficient to give a 1-form $\pi^{*} \theta$ on $X^{*}$ of homogeneity 1. This can be defined by $\pi^{*} \theta=i(\xi) \omega_{h}$, where $\xi \in H^{0}\left(\mathcal{M}, T_{\mathcal{M}}\right)$ is the holomorphic vector field generated by the $\mathbf{C}^{*}$ action. The non-degeneracy condition (1i) is exactly equivalent to requiring that the closed holomorphic 2 form $\omega_{h}$ satisfy $\omega_{h}^{n} \neq 0$. This is the case as $\omega_{h}$ is a holomorphic symplectic form.

The result follows. 
We will be able to determine the line orbibundle $L_{Z}$ explicitly. For this, consider the Hitchin map $\chi: \mathcal{M} \rightarrow \mathbf{C}^{3 g-3}$. As it is equivariant with respect to the $\mathbf{C}^{*}$ action, $\chi$ induces a map

$$
\bar{\chi}: Z \rightarrow \mathbf{P}^{3 g-4}
$$

on Z. The generic fibre of this map is easily seen to be the Kummer variety corresponding to the Prym variety (the Kummer variety of an Abelian variety is the quotient of the Abelian variety by the involution $x \rightarrow-x$ ), the generic fibre of the Hitchin map. Thus we have proved

Lemma 6.10 There exists a map $\chi: Z \rightarrow \mathbf{P}^{3 g-4}$ the reduction of the Hitchin map onto $Z$, for which the generic fibre is a Kummer variety.

Remark. This observation was already implicit in Oxbury's thesis (cf. 2.17a of Oxb]).

The following theorem determines the line bundle $L_{Z}$ in terms of the Hitchin map.

Theorem 6.11 $L_{Z}^{2}=\bar{\chi}^{*} \mathcal{H}_{3 g-4}$ where $\mathcal{H}_{3 g-4}$ is the hyperplane bundle on $\mathbf{P}^{3 g-4}$.

Proof. We understand from Corollary 6.7 that $\bar{\chi}^{*} \mathcal{H}_{3 g-4}=\mathcal{L}_{Z}^{k} \otimes L_{Z}^{l}$ for some integers $k$ and $l$.

We show that $k=0$. For this consider the pullback of $\mathcal{L}_{Z}$ onto $\mathcal{M} \backslash N$ the total space of the $\mathbf{C}^{*}$ principal orbibundle $X_{Z}^{*}$. This line orbibundle extends to $\mathcal{M}$ as $\mathcal{L}_{\mathcal{M}}$ and restricts to $T_{\mathcal{N}}^{*}$ as the pullback of $\mathcal{L}_{P T_{\mathcal{N}}^{*}}$ by construction. $c_{1}\left(\mathcal{L}_{\mathcal{M}}\right)$ is not trivial when restricted to $\mathcal{N}$ (namely it is $c_{1}\left(\mathcal{L}_{\mathcal{N}}\right)$, since this bundle is ample) therefore is not trivial when resticted to a generic fibre of the Hitchin map. We can deduce that $c_{1}\left(\mathcal{L}_{Z}\right)$ is not trivial on the generic fibre of $\bar{\chi}$.

However $L_{Z}$ restricted to a generic fiber of $\bar{\chi}$ can be described as follows. Let this Kummer variety be denoted by $K$, the corresponding Prym variety by $P$. Form the space $P \times \mathbf{C}^{*}$, the trivial $\mathbf{C}^{*}$ principal bundle on $P$ and quotient it out by the involution $\tau(p, z)=(-p,-z)$. The resulting space is easily seen to be the $\mathbf{C}^{*}$ orbit of the Prym $P$ in $\mathcal{M}$, therefore the total space of the $\mathbf{C}^{*}$ principal orbibundle $L_{Z}^{*} \backslash\left(L_{Z}^{*}\right)_{0}$ on $K$. Hence $L_{Z}^{2}$ is the trivial line orbibundle on $K$. Thus $c_{1}\left(\left.L_{Z}\right|_{K}\right)=0$.

Now $\bar{\chi}^{*} \mathcal{H}_{3 g-4}$ is trivial on the Kummer variety. Hence the assertion $k=0$.

The rest of the proof will follow the lines of Hitchin's proof of Theorem 6.2 in [Hit2]. We show that $l=2$.

The sections of $L_{Z}$ can be identified with holomorphic functions homogeneous of degree 2 on the $\mathbf{C}^{*}$ principal orbibundle $X_{Z}=L_{Z}^{*} \backslash\left(L_{Z}^{*}\right)_{0}$. As $N$ is of codimension $\geq 2$ such functions extend to $\mathcal{M}$. Since the Hitchin map is proper, these functions are constant on the fibers of the Hitchin map, therefore are the pullbacks of holomorphic functions on $\mathbf{C}^{3 g-3}$ of homogeneity 1 which can be identified with the holomorphic sections of the hyperplane bundle $\mathcal{H}_{3 g-4}$ on $P\left(\mathbf{C}^{3 g-3}\right)=\mathbf{P}^{3 g-4}$. 
Corollary 6.12 If $n$ is odd, there are natural isomorphisms

$$
H^{0}\left(Z, L_{Z}^{n}\right) \cong H^{0}\left(\mathcal{N}, S^{n} T_{\mathcal{N}}\right) \cong 0
$$

whereas if $n$ is even, then

$$
H^{0}\left(Z, L_{Z}^{n}\right) \cong H^{0}\left(\mathcal{N}, S^{n} T_{\mathcal{N}}\right) \cong H^{0}\left(\mathbf{P}^{3 g-4}, \mathcal{H}_{3 g-4}^{\frac{n}{2}}\right)
$$

Proof. We show that $H^{0}\left(Z, L_{Z}\right) \cong H^{0}\left(\mathcal{N}, S^{n}\left(T_{\mathcal{N}}\right)\right)$ for every $n$, the rest of the theorem will follow from Theorem 6.2 of [Hit2].

By Proposotion 6.3 we get that $H^{0}\left(Z, L_{Z}^{n}\right) \cong H^{0}\left(P T_{\mathcal{N}}^{*}, L_{P T_{\mathcal{N}}^{*}}^{n}\right)$. Let $\pi: P T_{\mathcal{N}}^{*} \rightarrow \mathcal{N}$ denote the projection. It is well known that the Leray spectral sequence for $\pi$ degenerates at the $E^{2}$ term. Moreover, we have that $R^{i} \pi_{*}\left(L_{P T_{\mathcal{N}}^{*}}^{n}\right)=0$ if $0<i<3 g-4$ (cf. Har] Theorem 5.1b). Therefore $H^{0}\left(P T_{\mathcal{N}}^{*}, L_{P T_{\mathcal{N}}^{*}}^{n}\right) \cong H^{0}\left(\mathcal{N}, \pi_{*}\left(L_{P T_{\mathcal{N}}^{*}}^{n}\right)\right)$. Finally the sheaf $\pi_{*}\left(L_{P T_{\mathcal{N}}^{*}}^{n}\right)$ is $S^{n}\left(T_{\mathcal{N}}\right)$, which proves the statement.

We can moreover determine the first cohomology group corresponding to the infintesimal deformations of the holomorphic contact structure on $Z$ and can interpret it in a nice way.

Corollary 6.13 There are canonical isomorphisms

$$
H^{1}\left(Z, L_{Z}\right) \cong\left(H^{1}\left(\mathcal{M}, \mathcal{O}_{\mathcal{M}}\right)\right)_{1} \cong H^{1}\left(\mathcal{N}, T_{\mathcal{N}}\right) \cong H^{1}\left(\Sigma, K_{\Sigma}^{-1}\right)
$$

where $\left(H^{1}\left(\mathcal{M}, \mathcal{O}_{\mathcal{M}}\right)\right)_{1} \subset H^{1}\left(\mathcal{M}, \mathcal{O}_{\mathcal{M}}\right)$ is the vector space of elements of $H^{1}\left(\mathcal{M}, \mathcal{O}_{\mathcal{M}}\right)$ homogeneous of degree 1 .

Proof. We may use the cohomological version of Hartog's theorem (cf. [Sche]) to show that $H^{1}\left(Z, L_{Z}\right) \cong H^{1}\left(P T_{\mathcal{N}}^{*}, L_{P T_{\mathcal{N}}^{*}}\right)$, as $Z$ and $P T_{\mathcal{N}}^{*}$ are isomorphic on an analytic set of codimension $\geq 3$ (cf. Proposition 6.3).

The proof of the other isomorphisms can be found in [Hit3].

Remark. We can interpret this result as saying that the deformation of the complex structure on $\Sigma$ corresponds to the deformation of complex structure on $\mathcal{N}$, to the deformation of holomorphic contact structure on $Z$ (cf. [Leb]) and to the deformation of the holomorphic symplectic structure of homogeneity 1 on $\mathcal{M}$.

As an easy corollary of the above we note the following

Corollary 6.14 The line orbibundle $L_{Z}$ is nef but neither trivial nor ample. 
Proof. $\quad L_{Z}$ is certainly not ample since $c_{1}\left(L_{Z}\right)$ is trivial on the Kummer variety.

On the other hand $L_{Z}^{2}$ being the pullback of an ample bundle is not trivial and is nef itself, hence the result.

The next theorem will describe the inherited Kähler structures of $Z$. Considering the one-parameter family of Kähler quotients $Q_{t}, t>c_{g-1}$ we get a one-parameter family of Kähler forms $\omega_{t}$ on $Z$. Theorem 1.1 from Du,He gives the following result for our case (cf. Theorem 3.3).

Theorem 6.15 (Duistermaat,Heckman) The complex orbifold $Z$ has a one-parameter family of Kähler forms $\omega_{t}, t>c_{g-1}$ such that

$$
\left[\omega_{t_{1}}(Z)\right]-\left[\omega_{t_{2}}(Z)\right]=\left(t_{1}-t_{2}\right) c_{1}\left(L_{Z}\right)
$$

where $t_{1}, t_{2}>c_{g-1}$ and $\left[\omega_{t}\right] \in H^{2}(Z, \mathbf{R})$ is the cohomology class of $\omega_{t}$.

Many of the above results will help us to prove the following theorem.

Theorem 6.16 $Z$ is a projective algebraic variety.

Proof. By the Kodaira embedding theorem for orbifolds (cf. [Bai]) we have only to show that $Z$ with a suitable Kähler form is a Hodge orbifold, i.e. the Kähler form is integer. For this to see we show that the Kähler cone of $Z$ contains a subcone, which is open in $H^{2}(Z, \mathbf{R})$. This is sufficient since such an open subcone should contain an integer Kähler form i.e. a Hodge form.

Since Corollary 6.7 shows that $\operatorname{Pic}_{0}(Z)$ is trivial, by Corollary 6.14 we see that $c_{1}\left(L_{Z}\right) \neq$ 0 . Therefore the previous theorem exhibited a half line in the Kähler cone of $Z$. Thus to find an open subcone in the 2 dimensional vector space $H^{2}(Z, \mathbf{R})$ (Corollary 6.7) it is sufficient to show that this line does not go through the origin or in other words $c_{1}(L)$ is not on the line. But this follows from Corollary 6.14, because $L$ being not ample $c_{1}(L)$ cannot contain a Kähler form. Hence the result.

Remark. We see from this proof that $c_{1}\left(L_{Z}\right)$ lies on the closure of the Kähler cone, thus $L_{Z}$ is nef. This reproves a statement of Corollary 6.14.

Example. In the case of the toy example the lowest level Kähler quotient $Z_{0}$ is the projectivized cotangent bundle $P T_{\mathcal{N}_{\text {toy }}}^{*}$ of $\mathcal{N}_{\text {toy }}$, which is isomorphic to $\mathcal{N}_{\text {toy }}=\mathbf{P}^{1}$, and the blowups and blowdowns add the four marked points to $\mathbf{P}^{1}$. Therefore $Z_{\text {toy }}$ is isomorphic to the orbifold $\mathbf{P}_{4}^{1}$, where the marked points correspond to the fixed point set of the involution $\sigma$, namely these are the projectivized bundles $P F_{i}$, i.e. points.

Moreover the $\mathbf{C}^{*}$ principal orbibundle $X_{Z_{\text {toy }}}$ on $\mathbf{P}_{4}^{1}$ has the form

$$
X_{Z_{\text {toy }}}=\left(P \times \mathbf{C}^{*}\right) /\left(\sigma_{P} \times \tau\right) \text {. }
$$

Thus in the toy example, not like in the ordinary Higgs case, we have $c_{1}\left(L_{Z_{\text {toy }}}\right)=0$. This latter assertion can be seen using 6.11 and noting that the target of the reduced toy Hitchin map $\bar{\chi}_{t o y}: Z_{t o y} \rightarrow \mathbf{P}^{0}$ is a point. 
There is an other difference, namely the Picard group of $Z_{\text {toy }}$ is of rank 1 , because $L_{Z_{\text {toy }}}^{2}$ is the trivial bundle on $Z_{\text {toy }}$.

In the next section we show how to compactify $\mathcal{M}$ by sewing in $Z$ at infinity.

\section{The compactification $\overline{\mathcal{M}}$}

In this section we compactify $\mathcal{M}$ by adding to each non-relatively compact $\mathbf{C}^{*}$ orbit an extra point i.e. sewing in $Z$ at infinity. Another way of saying the same is to glue together $\mathcal{M}$ and $E$ the total space of $L_{Z}$ along the $\mathbf{C}^{*}$ principal orbibundle $X_{Z}^{*}=E \backslash E_{0}=\mathcal{M} \backslash N$. To be more precise we use the construction of Lerman, called the symplectic cut (cf. Subsection 3.3 and [Ler]).

Since the complex structure on the Kähler quotients depends only on the connected component of the level, we can make the following definition.

Definition 7.1 Let $\overline{\mathcal{M}}_{d}$ denote the compact complex orbifold corresponding to the Kähler quotients of $\mathcal{M} \times \mathbf{C}$ by the product $S^{1}$ action

$$
\bar{M}_{\mu<t}=\left(\mu+\mu_{\mathbf{C}}\right)^{-1}(t) / S^{1},
$$

with $c_{d}<t<c_{d+1}$.

Let $X_{\overline{\mathcal{M}}_{d}}$ denote the corresponding $\mathbf{C}^{*}$ principal bundle on $\overline{\mathcal{M}}_{d}$. For simplicity we let $\overline{\mathcal{M}}$ denote $\overline{\mathcal{M}}_{g-1}$ and $X_{\overline{\mathcal{M}}}$ denote $X_{\overline{\mathcal{M}}_{g-1}}$.

As a consequence of the construction of symplectic cutting we have the following theorem (cf. Theorem 3.4)

Theorem 7.2 The compact orbifold $\overline{\mathcal{M}}=\mathcal{M} \cup Z$ is a compactification of $\mathcal{M}$ such that $\mathcal{M}$ is an open complex submanifold and $Z$ is a codimension one suborbifold, i.e. a divisor.

Moreover $\mathbf{C}^{*}$ acts on $\overline{\mathcal{M}}$ extending the action on $\mathcal{M}$ with the points of $Z$ being fixed.

In addition to the above we see that we have another decomposition $\overline{\mathcal{M}}=N \cup E$ of $\overline{\mathcal{M}}$ into the nilpotent cone and the total space $E$ of the contact line bundle $L_{Z}$ on $Z$. Thus the compactification by symplectic cutting produced the same orbifold as the two constructions we started this section with.

We start to list the properties of $\overline{\mathcal{M}}$. We will mention properties analogous to properties of $Z$ (these correspond to the fact that both spaces were constructed by a Kähler quotient procedure) and we will clarify the relation between $Z$ and $\overline{\mathcal{M}}$.

Theorem 3.4 and Theorem 3.3 give the following result in our case.

Theorem 7.3 $\overline{\mathcal{M}}_{d}$ is a compact orbifold. It has a decomposition $\overline{\mathcal{M}}_{d}=\mathcal{M}_{d} \cup Z_{d}$ into an open complex suborbifold $\mathcal{M}_{d}$ (which is actually a complex manifold) and a codimension one suborbifold $Z_{d}$, i.e. a divisor. The singular locus of $\overline{\mathcal{M}}_{d}$ coincides with that of $Z_{d}$ :

$$
\operatorname{sing}\left(\overline{\mathcal{M}}_{d}\right)=\operatorname{sing}\left(Z_{d}\right)=\bigcup_{0<i \leq d} P\left(F_{i}\right)
$$


where $F_{i}$ is a component of the fixed points set of the involution $\sigma(E, \Phi)=(E,-\Phi)$.

Furthermore, the $\mathbf{C}^{*}$ action on $\mathcal{M}_{d}$ extends onto $\overline{\mathcal{M}}_{d}$ with an extra component $Z_{d}$ of the fixed point set.

We have the corresponding statement of Theorem 6.4.

Theorem $7.4 \overline{\mathcal{M}}=\overline{\mathcal{M}}_{g-1}$ is birationally isomorphic to $\overline{\mathcal{M}}_{0}=P\left(T_{\mathcal{N}}^{*} \oplus \mathcal{O}_{\mathcal{N}}\right)$. Moreover, they are isomorphic outside an analytic subset of codimension at least 3.

Proof. In a similar manner to the proof of Corollary 6.4 we can argue by noting that $X_{\overline{\mathcal{M}}_{0}}$ is obviously isomorphic to $T_{\mathcal{N}}^{*} \oplus \mathcal{O}_{\mathcal{N}}$ with the standard action of $\mathbf{C}^{*}$. Hence indeed $\mathcal{M}_{0}=P\left(T_{\mathcal{N}}^{*} \oplus \mathcal{O}_{\mathcal{N}}\right)$.

By Theorem 3.3 it is clear that $\overline{\mathcal{M}}$ and $\overline{\mathcal{M}}_{0}$ are related by a sequence of blowups and blowdowns. The codimensions of the submanifolds we apply the blowups are at least 3 by a calculation analogous to the one in the proof of Proposition 6.3 .

Notation 7.5 Let

- $\mathcal{L}_{P\left(T_{\mathcal{N}}^{*} \oplus \mathcal{O}_{\mathcal{N}}\right)}$ denote the pull back of $\mathcal{L}_{\mathcal{N}}$ to $P\left(T_{\mathcal{N}}^{*} \oplus \mathcal{O}_{\mathcal{N}}\right)$,

- $\mathcal{L}_{\overline{\mathcal{M}}}$ be the corresponding line bundle on $\overline{\mathcal{M}}$.

- $L_{P\left(T_{\mathcal{N}}^{*} \oplus \mathcal{O}_{\mathcal{N}}\right)}$ be the dual of the tautological line bundle on the projective bundle $P\left(T_{\mathcal{N}}^{*} \oplus\right.$ $\left.\mathcal{O}_{\mathcal{N}}\right)$

- $L_{\overline{\mathcal{M}}}=X_{\overline{\mathcal{M}}} \times_{\mathbf{C}^{*}} \mathbf{C}$ be the corresponding line orbibundle on $\overline{\mathcal{M}}$.

Corollary 7.6 $\operatorname{Pic} \overline{\mathcal{M}}$ is isomorphic to $\operatorname{Pic}\left(P\left(T_{\mathcal{N}}^{*} \oplus \mathcal{O}_{\mathcal{N}}\right)\right)$ and therefore is of rank 2 and freely generated by $L_{\overline{\mathcal{M}}}$ and $\mathcal{L}_{\overline{\mathcal{M}}}$.

Proof. The previous theorem shows that $\overline{\mathcal{M}}$ and $P\left(T_{\mathcal{N}}^{*} \oplus \mathcal{O}_{\mathcal{N}}\right)$ are isomorphic outside an analytic subset of codimension at least 2, thus their Picard groups are naturally isomorphic.

However, $\operatorname{Pic}\left(P\left(T_{\mathcal{N}}^{*} \oplus \mathcal{O}_{\mathcal{N}}\right)\right)$ is freely generated by $L_{P\left(T_{\mathcal{N}}^{*} \oplus \mathcal{O}_{\mathcal{N}}\right)}$ and $\mathcal{L}_{P\left(T_{\mathcal{N}}^{*} \oplus \mathcal{O}_{\mathcal{N}}\right)}$. The result follows.

Corollary 7.7 The canonical line orbibundle $K_{\overline{\mathcal{M}}}$ of $\overline{\mathcal{M}}$ coincides with $L_{\overline{\mathcal{M}}}^{-(3 g-2)}$. Moreover, $L_{\overline{\mathcal{M}}}$ is the line bundle of the divisor $Z$, therefore $(3 g$-2) $Z$ is the anticanonical divisor of $\overline{\mathcal{M}}$. Finally, $L_{\overline{\mathcal{M}}}$ restricts to $L_{Z}$ to $Z$. 
Proof. $\quad L_{\overline{\mathcal{M}}}$ by its construction clearly restricts to $L_{Z}$ on $Z$ and it is the line bundle of $Z$, as the corresponding statement is obviously true for $P\left(T_{\mathcal{N}}^{*} \oplus \mathcal{O}_{\mathcal{N}}\right)$.

The restriction of $K_{\overline{\mathcal{M}}}$ to $\mathcal{M}$ has a non-zero section, namely the holomorphic Liouville form $\omega_{h}^{3 g-3}$, thus trivial. Hence $K_{\overline{\mathcal{M}}}=L_{\overline{\mathcal{M}}}^{k}$ for some $k \in \mathbf{Z}$.

By the second adjunction formula $K_{Z}=\left.\left(K_{\overline{\mathcal{M}}} \otimes[Z]\right)\right|_{Z}$. The right hand side equals $L_{Z}^{-(3 g-3)}$ as $L_{Z}$ is a contact line bundle (cf. 6.9). The left hand side can be written as $\left.\left(L_{\overline{\mathcal{M}}}^{k} \otimes L_{\overline{\mathcal{M}}}\right)\right|_{Z}=L_{Z}^{k+1}$, therefore $k=-(3 g-2)$.

Lemma $7.8 \chi$ has an extension to $\overline{\mathcal{M}}$,

$$
\bar{\chi}: \overline{\mathcal{M}} \rightarrow \mathbf{P}^{3 g-3}
$$

such that $\bar{\chi}$ restricted to $Z$ gives the map of Lemma 6.10 .

Proof. We let $\mathbf{C}^{*}$ act on $\mathbf{C}^{3 g-3} \times \mathbf{C}$ by $\lambda(x, z)=\left(\lambda^{2} x, \lambda z\right)$. With respect to this action the map $\left(\chi, i d_{\mathbf{C}}\right): \mathcal{M} \times \mathbf{C} \rightarrow \mathbf{C}^{3 g-3} \times \mathbf{C}$ is equivariant. Therefore making the symplectic cut it reduces to a map $\bar{\chi}: \overline{\mathcal{M}} \rightarrow \mathbf{P}^{3 g-3}$ since the quotient space $\left(\mathbf{C}^{3 g-3} \backslash 0\right) \times \mathbf{C} / \mathbf{C}^{*}$ is isomorphic to $\mathbf{P}^{3 g-3}$.

The result follows.

Remark. In the higher rank case where $\mathbf{C}^{*}$ acts on the target space of the Hitchin map with different weights the target space of the compactified Hitchin map is a weighted projective space.

Corollary $7.9 L_{\overline{\mathcal{M}}}^{2}=\bar{\chi}^{*} \mathcal{H}_{3 g-3}$.

Proof. Obviously, $\left.\bar{\chi}^{*} \mathcal{H}_{3 g-3}\right|_{\mathcal{M}}$ is trivial, therefore $\bar{\chi}^{*} \mathcal{H}_{3 g-3}$ is some power of $L_{\overline{\mathcal{M}} \text {. By }}$ 6.11 this power is 2 .

Theorem 7.10 (Duistermaat, Heckman) $\overline{\mathcal{M}}$ has a one-parameter family of Kähler forms $\omega_{t}(\overline{\mathcal{M}}), t>c_{g-1}$ such that

$$
\left[\omega_{t_{1}}(\overline{\mathcal{M}})\right]-\left[\omega_{t_{2}}(\overline{\mathcal{M}})\right]=\left(t_{1}-t_{2}\right) c_{1}\left(L_{\overline{\mathcal{M}}}\right) .
$$

Furthermore this one-parameter family of Kähler forms restricts to $Z$ as the oneparameter family of Kähler forms of Theorem 6.15.

Proof. This is just the application of Theorem 3.3 and Theorem 3.4 to our situation.

Corollary $7.11 \overline{\mathcal{M}}$ is a projective algebraic variety. 
Proof. The argument is the same as for Theorem 6.16, noting that by Corollary 7.6 $H^{2}(\overline{\mathcal{M}}, \mathbf{R})$ is two dimensional and $L_{\overline{\mathcal{M}}}$ is neither trivial nor ample since $L_{\overline{\mathcal{M}}} \mid Z=L_{Z}$ (by Corollary 7.7) is neither trivial nor ample (by Corollary 6.14).

Remark. 1. The above proof yields that the cohomology class $c_{1}\left(L_{\overline{\mathcal{M}}}\right)$ sits in the closure of the Kähler cone of $\overline{\mathcal{M}}$, hence $L_{\overline{\mathcal{M}}}$ is nef.

2. From the previous remark and Corollary 7.9 we can deduce that there is a complete hyperkähler (hence Ricci flat) metric on $\overline{\mathcal{M}}=\overline{\mathcal{M}} \backslash Z$, the complement of a nef anticanonical divisor of a compact orbifold.

Therefore our compactification of $\mathcal{M}$ is compatible with Yau's problem, which addresses the question: which non-compact complex manifolds possess a complete Ricci flat metric? Tian and Yau in Ti,Ya could show that this is the case for the complement of an ample anticanonical divisor in a compact complex manifold. (Such manifolds are called Fano manifolds.)

The similar statement with ample replaced by nef is an unsolved problem.

\section{Theorem $7.12 \overline{\mathcal{M}}$ has Poincaré polynomial}

$$
P_{t}(\overline{\mathcal{M}})=P_{t}(\mathcal{M})+t^{2} P_{t}(Z)
$$

Proof. We have three different ways of calculating the Poincaré polynomial of $\overline{\mathcal{M}}$. The first is through Kirwan's formula in [Kir], the second is due to Thaddeus in [Tha3], which we used to calculate the Poincaré polynomial of $Z$.

For $\overline{\mathcal{M}}$ there is a third method, namely direct Morse theory. All we have to note is that the $S^{1}$ action $\overline{\mathcal{M}}$ is Hamiltonian with respect to any Kähler form of Theorem 7.10 , and the critical submanifolds and corresponding indices are the same as for $\mathcal{M}$ with one extra critical submanifold $Z$ of index 2 . Hence the result.

Example. We can describe $\overline{\mathcal{M}}_{\text {toy }}=\mathcal{M}_{\text {toy }} \cup Z_{\text {toy }}$ as follows. As we saw above $\mathcal{M}_{\text {toy }} \backslash N_{\text {toy }}=$ $X_{Z_{\text {toy }}}$. Thus gluing together $\mathcal{M}_{\text {toy }}$ and $E_{\text {toy }}$, the total space of the line orbibundle $L_{Z_{\text {toy }}}$, along $X_{Z_{\text {toy }}}$ yields

$$
\overline{\mathcal{M}}_{\text {toy }}=\mathcal{M}_{\text {toy }} \cup_{X_{Z_{\text {toy }}}} E_{\text {toy }} .
$$

One can construct $\overline{\mathcal{M}}_{\text {toy }}$ directly, as follows. Take $\mathbf{P}^{1}=\mathbf{C} \cup \infty$ extending the involution $\tau$ from $\mathbf{C}$ to $\mathbf{P}^{1}$. Consider the quotient $\left(P \times \mathbf{P}^{1}\right) /\left(\sigma_{P} \times \tau\right)$. This is a compact orbifold with eight $\mathbf{Z}_{2}$-quotient singularities. Blow up four of them corresponding to $0 \in \mathbf{C}$. The resulting space will be isomorphic to $\overline{\mathcal{M}}_{\text {toy }}$. The remained four isolated $\mathbf{Z}_{2}$ quotient singularities will just be the four marked points of $Z_{\text {toy }} \subset \overline{\mathcal{M}}_{\text {toy }}$, the singular locus of $\overline{\mathcal{M}}_{\text {toy }}$.

We finish this section with a result which gives an interesting relation between the intersections of the component of the nilpotent cone $N$ in $\mathcal{M}$ (equivalently the intersection 
form on the middle compact cohomology $H_{c p t}^{6 g-6}(\mathcal{M})$, cf. Corollary 5.4) and the contact structure of $Z$.

Theorem 7.13 There is a canonical isomorphism between the cokernel of $j_{\mathcal{M}}$ and the cokernel of $L$, where

$$
j_{\mathcal{M}}: H_{c p t}^{6 g-6}(\mathcal{M}) \rightarrow H^{6 g-6}(\mathcal{M})
$$

is the canonical map and

$$
L: H^{6 g-8}(Z) \rightarrow H^{6 g-6}(Z)
$$

is multiplication with $c_{1}\left(L_{Z}\right)$.

Proof. We will read off the statement from the following diagram.

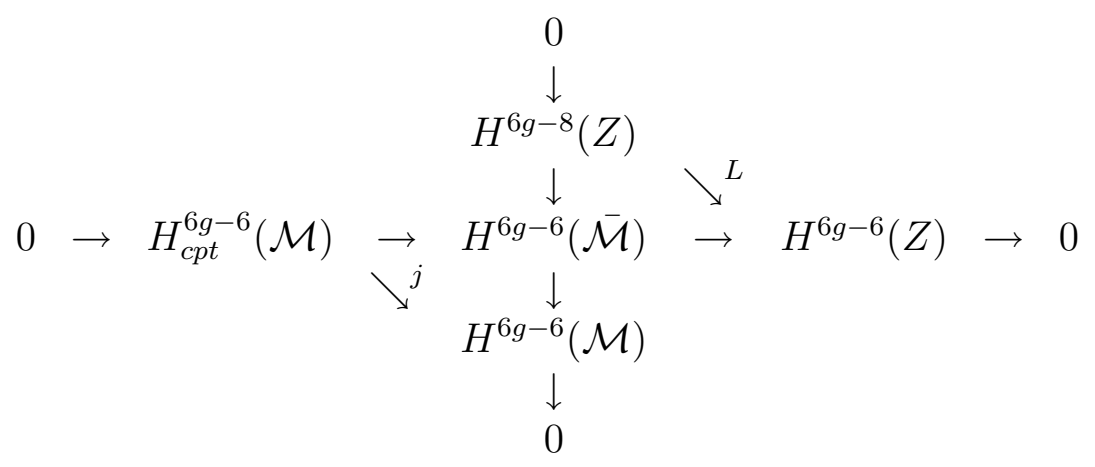

We show that both the vertical and horizontal sequences are exact and the two triangles commute.

From the Bialynicki-Birula decomposition of $\overline{\mathcal{M}}$ we get the short exact sequence of middle dimensional cohomology groups (recall that $E \subset \overline{\mathcal{M}}$ denotes the total space of the contact line bundle $L_{Z}$ on Z):

$$
0 \rightarrow H_{c p t}^{6 g-6}(E) \rightarrow H^{6 g-6}(\overline{\mathcal{M}}) \rightarrow H^{6 g-6}(\mathcal{M}) \rightarrow 0 .
$$

Applying the Thom isomorphism (which also exists in the orbifold category) we can identify $H_{c p t}^{6 g-6}(E)$ with $H^{6 g-8}(Z)$, this gives the vertical short exact sequence of the diagram. The horizontal one is just its dual short exact sequence.

Finally, the left triangle clearly commutes as all the maps are natural, while the right triangle commutes because the original triangle commuted as above and the canonical map $j_{E}: H_{c p t}^{6 g-6}(E) \rightarrow H^{6 g-6}(E)$ transforms to $L: H^{6 g-8}(Z) \rightarrow H^{6 g-6}(Z)$ by the Thom isomorphism.

Now the theorem is the consequence of the Butterfly lemma (cf. Lan IV.4 p.102), or can be proved by an easy diagram chasing.

Hence the result follows.

Remark. 1. If the line bundle $L_{Z}$ was ample then the map $L$ would just be the Lefschetz isomorphism, and therefore the cokernel would be trivial. In our case we have $L_{Z}$ being only nef and the map is not an isomorphism, the cohomology class of the Kummer variety lying in the kernel. Therefore the cokernel measures how far is $L_{Z}$ from being ample. 
2. The cokernel of $j_{\mathcal{M}}$ measures the degeneracy of the intersection form on the compactly supported middle dimensional cohomology of $\mathcal{M}$. In this case also the cokernel is not trivial as the compactly supported cohomology class of the Prym variety lies in the kernel. This can be seen by thinking of the Hitchin map as a section of the trivial rank $3 g-3$ vector bundle on $\mathcal{M}$ and considering the ordinary cohomology class of the Prym variety as the Euler class of this trivial vector bundle, and as such, the ordinary cohomology class of the Prym variety is trivial indeed.

3. Notice that the proof did not use any particular property of $\mathcal{M}$ therefore the statement is true in the general setting of Section 3 .

Example. 1. We determine the dimension of the cokernels of the above theorem in the case when $g=2$, by showing that the intersection form on the compactly supported middle dimensional cohomology is 0 , i.e. the map $j_{\mathcal{M}}$ is zero. In the previous remark we saw that the compactly supported cohomology class of the Prym variety $P$ is in the kernel of $j_{\mathcal{M}}$. It follows from Tha1 that the compactly supported cohomology of $\mathcal{N}$ and that of $P$ generates the 2-dimensional compactly supported middle cohomology of $\mathcal{M}$ (cf. Theorem 5.4).

On the other hand the Euler characteristic of $\mathcal{N}$ is 0 (this can be checked by substituting -1 in the known Poincaré polynomial of $\mathcal{N}$, see e.g. At,Bo ), so the Euler class of $T_{\mathcal{N}}^{*}$ vanishes. Therefore $\mathcal{N}$ has self intersection number 0 in $T_{\mathcal{N}}^{*} \subset \mathcal{M}$. This shows that the intersection form is zero.

2. We can also calculate the dimension of the cokernels in our toy example. Namely, the dimension of $\operatorname{coker}\left(L_{\text {toy }}\right)$ is clearly 1 , as the map $L_{\text {toy }}: H^{0}\left(Z_{\text {toy }}\right) \rightarrow H^{2}\left(Z_{\text {toy }}\right)$ is the multiplication with $c_{1}\left(L_{Z_{\text {toy }}}\right)=0$ (cf. the example at the end of Section 6).

Thus, by the above theorem, we have that $\operatorname{coker}\left(j_{\mathcal{M}_{\text {toy }}}\right)$ is 1 -dimensional. It can be seen directly, using Zariski's lemma (Lemma 8.2 in [B,P,V] p. 90), that the kernel of the map $j_{\mathcal{M}_{\text {toy }}}$ is generated by the cohomology class of the elliptic curve $P$, the generic fibre of the toy Hitchin map, hence it is 1 dimensional, indeed.

\section{References}

[At,Bo] M. Atiyah, R. Bott. The Yang-Mills equations over Riemann surfaces. Philos. Trans. Roy. Soc. London Series A, 308 (1982) 523-615

[Bai] W.L. Bailey, Jr. On the embedding of V-manifolds in projective space. Americal Journal of Mathematics, 79 (1957) 403-430,

[B,P,V] W. Barth, C. Peters, A. Van de Van. Compact complex surfaces, Springer Verlag, 1984

[Bia] A. Bialynicki-Birula. Some theorems on actions of algebraic groups, Ann. of Math.98 (1973) 480-497

[Bi,Da] R. Bielawski, A.S. Dancer, The geometry and topology of toric hyperkähler manifolds. Preprint, 1996 
[Br,Pr] M. Brion, C. Procesi, Action d'un tore dans une variété projective, Operator algebras, unitary representations, enveloping algebras, and invariant theory (A.Connes, M.Duflo, A. Joseph, and R.Rentschler, eds.), Birkhauser, (1990), pp. 509-539

[Dr,Na] J.-M. Drezet, M.S. Narasimhan, Groupe Picard des variétés de modules de fibrés semistables sur les courbes algébraiques, Invent. Math. 97 53-94 (1989)

[Du,He] J.J. Duistermaat, G.J. Heckman. On the variation in the cohomology of the symplectic form of the reduced phase space. Invent. Math. 69 (1982) 259-268

[Ed,Gr] D. Edidin, W. Graham, Algebraic cuts, Preprint, alg-geom/9608028

[Goth1] P.B. Gothen, The Betti numbers of the moduli space of rank 3 Higgs bundles, Internat. J. Math. 5 (1994) 861-875

[Goth2] Private communication

[Goto] R. Goto. On Toric hyperkähler manifolds given by hyperkähler quotient method, in 'Infinite Analysis', Advanced Series in Mathematical Physics 16 (1992) 317388

[Gr,Ha] P. Griffiths, J. Harris. Principles of algebraic geometry. New York, Wiley 1978

[Gu,St] V. Guillemin, S. Sternberg, Birational equivalences in the symplectic category. Invent. Math 97, (1989) 485

[Har] R. Hartshorne, Algebraic geometry Springer-Verlag, New York 1977

[Hit1] N. Hitchin. The self-duality equations on a Riemann surface, Proc. London Math. Soc. (3) 55 (1987) 59-126.

[Hit2] N. Hitchin. Stable vector bundles and integrable systems, Duke Mathematical Journal (1) 54 (1987) 91-114

[Hit3] N. Hitchin. Flat connections and geometric quantization, Comm. Math. Phys. 131, (1990) 347-380

[Kir] F.C. Kirwan. Cohomology of quotients in symplectic and algebraic geometry, Mathematical Notes 31, Princeton University Press, 1984

[Lan] S. Lang. Algebra Addison-Wesley, Reading, Massachusetts, 1965

[Ler] E. Lerman. Symplectic Cuts, Math. Res. Lett. 2 (1995) 247-258

[Lau] G. Laumon. Un analogue du cone nilpotent. Duke Math. J. (2) 57 (1988) 647-671

[Leb] C. Lebrun. Fano manifolds, contact structures and quaternionic geometry, International Journal of Mathematics (3) 6 (1995) 419-437 
[Nak] H. Nakajima. Lectures on Hilbert schemes of points on surfaces. to appear

[Nit] N. Nitsure. Moduli space of semistable pairs on a curve. Proc. London Math. Soc. (3) 62 (1991), 275-300

[Sche] G. Scheja, Riemannsche Hebbarkeitsatze für cohomologieklassen, Math. Ann. 144 (1961), 345-360

[Schm] A. Schmitt. Projective moduli of Hitchin pairs, Preprint, alg-geom/9611008

[Sim1] C. Simpson. Higgs bundles and local systems. Publ. Math. I.H.E.S. 75 (1992), $5-95$

[Sim2] C. Simpson. The Hodge filtration on nonabelian cohomology, alg-geom 9604005

[Sim3] C. Simpson, Nonabelian Hodge Theory. Proceedings of the International Mathematical Congress, Kyoto, 1990, 747-756

[Tha1] M. Thaddeus. Topology of the moduli space of stable bundles over a compact Riemann surface, 1990, unpublished

[Tha2] M. Thaddeus. Stable pairs, linear systems and the Verlinde formula, Invent. Math. 117 (1994) 317-353

[Tha3] M. Thaddeus. Geometric invariant theory and flips, Journal of the American Mathematical Society (3) 9 (1996)

[Oxb] W. Oxbury. Stable bundles and branched coverings over Riemann surfaces. D.Phil thesis, Oxford, 1987

[Ti,Ya] G. Tian, S.H. Yau. Complete Kähler manifolds with zero Ricci curvature. I. J.Amer.Math.Soc. (3) 3 (1990) 579-609

[Yoko] K. Yokogawa. Compactification of moduli of parabolic sheaves and moduli of parabolic Higgs sheaves; J. Math. Kyoto Univ. 33 (1993), 451-504 